\title{
Reasons for Primary Medication Nonadherence: A Systematic Review and Metric Analysis
}

\author{
Shan-Qi Lee, BSocSc; Aravind Sesagiri Raamkumar, MSc; Jinhui Li, BEng; Yuanyuan Cao, JM; \\ Kanokkorn Witedwittayanusat, MA; Luxi Chen, PhD; and Yin-Leng Theng, PhD
}

\begin{abstract}
BACKGROUND: The behavior of medication nonadherence is distinguished into primary and secondary nonadherence. Primary nonadherence (PNA) is not as thoroughly studied as secondary nonadherence.
\end{abstract}

OBJECTIVE: To explore and synthesize contributing factors to PNA based on the existing body of literature.

METHODS: A search was performed on the PubMed, PsycINFO, CINAHL, and ScienceDirect databases to identify previously published scholarly articles that described the "factors," "reasons," "determinants" or "facilitators" of PNA. The alternate spelling of "non-adherence" was used as well. The effect that the articles had in the research community, as well as across social media, was also explored.

RESULTS: 22 studies met the inclusion criteria for this review. The PNA factors that the studies identified were diverse, spanning economic, social, and medical dimensions. A multilevel classification method was applied to categorize the factors into 5 broad groups-patient, medication, health care provider, health care system, and socioeconomic factors. Patient factors were reported the most. Some groups overlapped and shared a dynamic causal relationship where one group influenced the outcome of the other.

CONCLUSIONS: Like all nonadherence behaviors, PNA is multifaceted with highly varied contributing factors that are closely associated with one another. Given the multidimensional nature of PNA, future intervention studies should focus on the dynamic relationship between these factor groups for more efficient outcomes.

J Manag Care Spec Pharm. 2018;24(8):778-94

Copyright $\odot 2018$, Academy of Managed Care Pharmacy. All rights reserved.

\section{What is already known about this subject}

Primary nonadherence (PNA) is a subcategory of medication nonadherence and occurs when a patient fails to initiate treatment by not picking up prescribed medications.

Contributing factors to medication nonadherence are complex and multidimensional and can be broadly classified into 5 categories: patient, medication, health care provider, health care system, and socioeconomic factors.

PNA and its contributing factors are not as widely studied as secondary nonadherence (where a patient does not take medication as prescribed, fails to refill the prescription, or discontinues it altogether)

\section{What this study adds}

This study examined contributing factors to PNA through a systematic review, in which all factors were classified using a multilevel approach, and the relationships between each classification were explored and established.

This review incorporated article bibliometrics and altmetrics to provide insight on readership, impact, and outreach in the context of citations and social media.

Results from the review indicate that PNA is receiving more attention within the research community, as well as in social media and the news.

$\mathrm{M}$ edication adherence is how patients' behavior corresponds with the medication regimen prescribed or advised by their health care provider. ${ }^{1}$ Therefore, medication nonadherence denotes a "passive failure" to follow the recommended prescription and represents a multifaceted problem with widespread consequences on the patient and patient-physician relationship as well as health care plans and the health care system as whole. ${ }^{2}$ Medication nonadherence is classified into 2 main categories-primary and secondary nonadherence.

According to Adams and Stolpe (2016), primary medication nonadherence occurs when a new medication is issued to patients, but they fail to pick it up or initiate treatment within a stipulated time period. ${ }^{3}$ On the other hand, secondary medication nonadherence happens when patients do not take their medication as prescribed, do not refill the prescription on time, or discontinue their medication altogether., In the existing literature, there is little research on primary medication nonadherence (PNA), with most works focusing on secondary nonadherence. One main reason lies in the difficulty of capturing PNA, where measurement is contingent on prescription and dispensing information that is typically stored in different databases. ${ }^{6}$ However, neglecting PNA in the study of medication nonadherence leads to underestimating the overall nonadherence rate, as secondary nonadherence alone does not account for patients who do not pick up new medications. ${ }^{7}$ In addition, from a clinical standpoint, a patient who fails to initiate treatment poses a different problem from the patient who does so but falls short only at a later point. ${ }^{8}$ As 
such, it is important to gain greater insight into the rates and determinants of PNA in the study of medication nonadherence.

As posited by Dalvi and Mekoth (2017), there is no single factor for medication nonadherence. ${ }^{9}$ Factors informing PNA behaviors are a complex web of interactive causes spanning the economic, social, and medical dimensions, including patients' attitudes toward taking medication. Miller et al. (1997) broadly categorize nonadherence behaviors into patient factors, medication factors, health care provider factors, health care system factors, and socioeconomic factors. ${ }^{10}$ This categorization represents a multilevel and multidisciplinary approach that is critical in devising "suitable and individualized solutions" to improve adherence among patients, especially those with chronic conditions. ${ }^{1}$ As discussed, PNA has not been extensively studied, with few works that explore the contributing factors behind it. Given this background, a systematic review was therefore carried out to investigate the barriers to primary medication adherence worldwide.

\section{Methods}

\section{Selection Process}

This systematic review was conducted and reported using the PRISMA Statement. A literature search was performed on the PubMed, PsycINFO, CINAHL, and ScienceDirect databases to identify articles containing information on factors of PNA. The search was limited to English-language publications, covering the time period before March 2017 and excluding letters, editorials, and book chapters. Keywords were primary nonadherence, primary nonadherence, and primary medication adherence. As the topic of PNA has not been widely studied, associated keywords for factors were excluded to yield a larger pool of literature for the first-round search.

A flowchart of the literature search is presented in Figure 1. The first round identified 92 articles. After removing duplicates, 69 articles were further screened based on their titles and abstracts to determine their eligibility. Studies that cover both primary and secondary nonadherence were included, while those that only explore the general subject of nonadherence without differentiating primary and secondary were excluded.

The remaining 35 articles were then reviewed in detail in the second round of selection. Articles that examined "factors," "reasons," "determinants," or "facilitators" of PNA were retained, while those focusing only on rates or intervention methods were omitted. Articles were included in this review if (a) a dosing regimen for any medication was evaluated and PNA reported; (b) specific factors associated with PNA were identified and explored, either as a primary or secondary outcome of the study; (c) they were not interventions designed to improve adherence or test the effectiveness of intervention methods, or trials implemented to study the influence of a certain hypothesized factor; and (d) they described the study design and specified the measurement method and definition of PNA. Therefore, 22 scholarly articles met the final eligibility criteria and formed the basis of this systematic review.

\section{Metric Analysis}

We also analyzed the bibliometric and altmetric outreach of the articles with the aim of understanding the outreach and impact they have on research and society.

Bibliometrics are traditional, well-known impact indicators such as citation count, journal impact factor, or $h$-index. ${ }^{11}$ In the last few years, research has been increasingly disseminated and discussed on social media. Altmetrics refer to new or alternative metrics aimed at measuring the impact of research. ${ }^{12}$ Popular examples of altmetrics include tweet count on Twitter, Facebook post count, and Wikipedia mention count. Altmetrics offer a faster and real-time measure of impact when compared with bibliometrics. For instance, if a research article is mentioned across multiple tweets or discussed in Wikipedia pages and blog articles, this provides an indication of the topic's popularity across social media. Such instances also reflect the impact of research on a wider audience, moving beyond the research community. Hence, the reach and popular appeal of research articles can be ascertained by analyzing the almetric scores. However, altmetrics still face many data quality issues and are easier to manipulate when compared with traditional bibliometrics. ${ }^{13}$

On May 8, 2017, we collected tweet count, Mendeley readers count, and the Altmetric Attention Score from Altmetric. com, usage and capture data from PlumX, and citation count from Scopus for the articles in the review. Altmetrics are based mainly on social media data sources. The Altmetric Attention Score is an aggregate weighted metric based on data sources such as news outlets, policy documents, blogs, Wikipedia, Twitter, Facebook, and YouTube. PlumX provides usage data that comprise counts from article downloads, article views, library holdings, video plays, clicks, collaborators, and others. It also offers capture data that consist of counts from bookmarks, favorites, followers, readers, subscribers, viewers, exports, saves, and code forks. To identify citations from prestigious universities, we used the 2015 Quacquarelli Symonds (QS) university rankings. Therefore, the citations extracted from Scopus were classified either as QS or non-QS. The data visualizations are based on a logarithmic scale. Through this metric analysis, our intention is to provide additional insight on the readership, impact, popularity, and attention of PNA in the context of citations and social media networks. We believe that a review article with such metric data would help readers understand prior studies in a different perspective. For instance, further exploration of cited articles and articles shared on social media could provide cues on future research agendas and help readers to frame research questions. 


\section{FIGURE 1 Literature Search Flowchart}

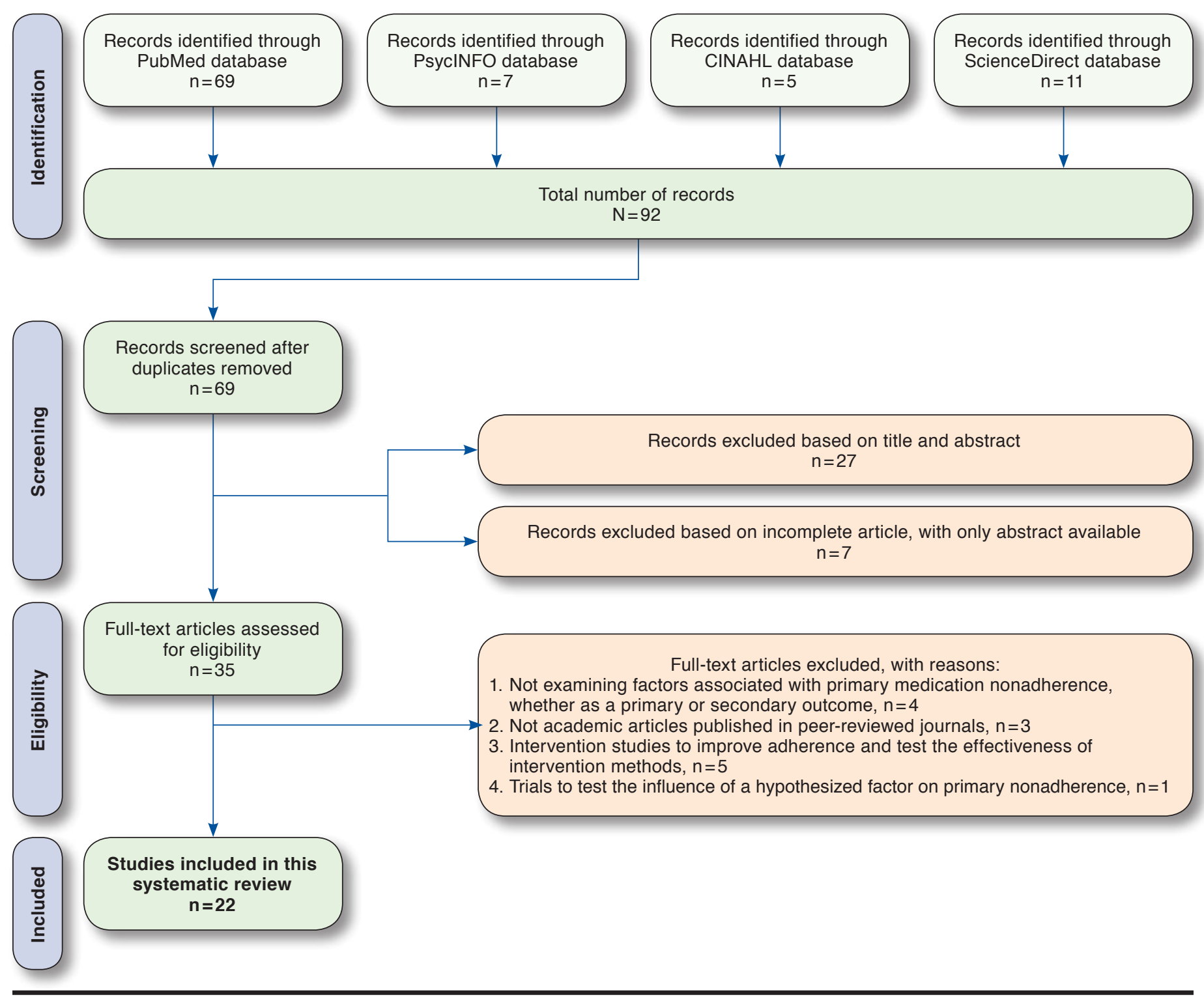

\section{Results}

Twenty-two articles met the eligibility criteria and were included in this systematic review. Details such as country of study, participant profile, methods used to assess adherence, and all associated factors of reported nonadherence are summarized in Table 1.

Factors of medication nonadherence, both primary and secondary, are complex and multifaceted. All 22 articles in this review addressed a range of reasons for PNA, covering factors across the economic, social, and medical spectrums. We further organized these reasons according to the multilevel classification system recommended by Miller et al. as shown in Table $1 .{ }^{10}$ Each article addresses at least 1 of the classified factors, as elaborated in the following brief descriptions.

\section{Patient Factors}

Of the 22 articles included in this systematic review, 19 addressed patient factors. These factors encompassed demographics, beliefs/perceptions/attitudes, physical and mental health conditions, medical history, and others. We found that patients' perceptions toward the effectiveness, risks, and necessity of their prescribed medication constituted a predominant 
TABLE 1 Studies Included in Systematic Review

\begin{tabular}{|c|c|c|c|c|c|c|c|c|c|}
\hline \multirow[b]{2}{*}{ Source } & \multirow[b]{2}{*}{ Country } & \multicolumn{2}{|c|}{ Participant Profile } & \multirow[b]{2}{*}{$\begin{array}{c}\text { How } \\
\text { Adherence Is } \\
\text { Assessed }\end{array}$} & \multicolumn{5}{|c|}{ Main Factors Addressed for Primary Nonadherence } \\
\hline & & $\begin{array}{l}\text { Final } \\
\text { Sample } \\
\text { Size }\end{array}$ & $\begin{array}{c}\text { Sample } \\
\text { Description }\end{array}$ & & $\begin{array}{l}\text { Patient } \\
\text { Factors }\end{array}$ & $\begin{array}{l}\text { Medication } \\
\text { Factors }\end{array}$ & $\begin{array}{l}\text { Health Care } \\
\text { Provider } \\
\text { Factors }\end{array}$ & $\begin{array}{l}\text { Health Care } \\
\text { System } \\
\text { Factors }\end{array}$ & $\begin{array}{l}\text { Socioeconomic } \\
\text { Factors }\end{array}$ \\
\hline $\begin{array}{l}\text { Adamson } \\
\text { et al. }(2017)^{14}\end{array}$ & $\begin{array}{l}\text { United } \\
\text { States }\end{array}$ & 2,496 & $\begin{array}{l}\text { New dermatol- } \\
\text { ogy patients } \\
\text { with } 1 \text { or more } \\
\text { medications } \\
\text { prescribed }\end{array}$ & $\begin{array}{l}\text { Electronic } \\
\text { medical records } \\
\text { and pharmacy } \\
\text { claims records }\end{array}$ & $\begin{array}{l}\text { Higher PNA } \\
\text { among younger } \\
\text { patients } \\
\text { Age factor } \\
\text { differs in men } \\
\text { and women }\end{array}$ & $\begin{array}{l}\text { Number of } \\
\text { dispensed drug } \\
\text { (polypharmacy) } \\
\text { Cost, especially } \\
\text { for elderly } \\
\text { patients }\end{array}$ & - & $\begin{array}{l}\text { Method of } \\
\text { prescription } \\
\text { (electronic } \\
\text { prescription } \\
\text { vs. paper } \\
\text { prescription) } \\
\text { Infrastructure } \\
\text { to accommo- } \\
\text { date the needs } \\
\text { of non-English- } \\
\text { speaking } \\
\text { patients }\end{array}$ & - \\
\hline $\begin{array}{l}\text { Bauer } \\
\text { et al. }(2013)^{22}\end{array}$ & $\begin{array}{l}\text { United } \\
\text { States }\end{array}$ & 1,366 & $\begin{array}{l}\text { Adults aged } \\
30-75 \text { years } \\
\text { with type } 2 \\
\text { diabetes who } \\
\text { were prescribed } \\
\text { a new } \\
\text { antidepressant } \\
\text { during } \\
2006-2010\end{array}$ & $\begin{array}{l}\text { Pharmacy } \\
\text { claims records }\end{array}$ & $\begin{array}{l}\text { Health literacy } \\
\text { and race/ } \\
\text { ethnicity }\end{array}$ & - & - & - & $\begin{array}{l}\text { Patients with } \\
\text { health literacy } \\
\text { limitations } \\
\text { have poorer } \\
\text { adherence }\end{array}$ \\
\hline $\begin{array}{l}\text { Cheetham } \\
\text { et al. }(2013)^{15}\end{array}$ & $\begin{array}{l}\text { United } \\
\text { States }\end{array}$ & 19,826 & $\begin{array}{l}\text { Patients aged } \\
\geq 24 \text { years with } \\
\text { a new statin } \\
\text { prescription } \\
\text { (having no } \\
\text { statin prescrip- } \\
\text { tions in the } \\
\text { previous } 12 \\
\text { months) in a } \\
\text { large integrated } \\
\text { health care } \\
\text { delivery system }\end{array}$ & $\begin{array}{l}\text { Electronic } \\
\text { medical records }\end{array}$ & $\begin{array}{l}\text { Younger and } \\
\text { healthier } \\
\text { patients, with } \\
\text { fewer comorbid } \\
\text { conditions, } \\
\text { lower rates of } \\
\text { hospitalization, } \\
\text { fewer clinic } \\
\text { and emergency } \\
\text { department } \\
\text { visits in } \\
\text { previous year }\end{array}$ & \begin{tabular}{|l|} 
Fewer \\
concurrent \\
prescriptions
\end{tabular} & - & - & - \\
\hline $\begin{array}{l}\text { da Costa } \\
\text { et al. }(2015)^{20}\end{array}$ & Portugal & 375 & $\begin{array}{l}\text { Patients aged } \\
\geq 15 \text { years with } \\
\text { chronic medical } \\
\text { conditions and } \\
\text { a prescription } \\
\text { of at least } \\
1 \text { drug for } \\
\text { diabetes, } \\
\text { hypertension, } \\
\text { or } \\
\text { hyperlipidemia }\end{array}$ & $\begin{array}{l}\text { Questionnaire } \\
\text { study and data } \\
\text { of medication } \\
\text { collection from } \\
\text { pharmacist }\end{array}$ & $\begin{array}{l}\text { Higher nonad- } \\
\text { herence among } \\
\text { women for } \\
\text { antidiabetic } \\
\text { medication }\end{array}$ & $\begin{array}{l}\text { Availability of } \\
\text { medication at } \\
\text { home } \\
\text { Higher nonad- } \\
\text { herence among } \\
\text { women for } \\
\text { antidiabetic } \\
\text { medication }\end{array}$ & - & - & $\begin{array}{l}\text { Financial } \\
\text { problems faced } \\
\text { by patients }\end{array}$ \\
\hline $\begin{array}{l}\text { Fallis } \\
\text { et al. }(2013)^{33}\end{array}$ & Canada & 232 & $\begin{array}{l}\text { Patients aged } \\
\geq 66 \text { years, dis- } \\
\text { charged from } \\
\text { the general } \\
\text { internal medi- } \\
\text { cine service of } \\
\text { a hospital }\end{array}$ & $\begin{array}{l}\text { Claims data in } \\
\text { Drug Profile } \\
\text { Viewer }\end{array}$ & - & - & $\begin{array}{l}\text { More likely } \\
\text { among patients } \\
\text { discharged to } \\
\text { nursing homes }\end{array}$ & - & - \\
\hline
\end{tabular}




\section{TABLE 1 Studies Included in Systematic Review (continued)}

\begin{tabular}{|c|c|c|c|c|c|c|c|c|c|}
\hline \multirow[b]{2}{*}{ Source } & \multirow[b]{2}{*}{ Country } & \multicolumn{2}{|c|}{ Participant Profile } & \multirow[b]{2}{*}{$\begin{array}{c}\text { How } \\
\text { Adherence Is } \\
\text { Assessed }\end{array}$} & \multicolumn{5}{|c|}{ Main Factors Addressed for Primary Nonadherence } \\
\hline & & $\begin{array}{l}\text { Final } \\
\text { Sample } \\
\text { Size }\end{array}$ & $\begin{array}{c}\text { Sample } \\
\text { Description }\end{array}$ & & $\begin{array}{l}\text { Patient } \\
\text { Factors }\end{array}$ & $\begin{array}{l}\text { Medication } \\
\text { Factors }\end{array}$ & $\begin{array}{l}\text { Health Care } \\
\text { Provider } \\
\text { Factors }\end{array}$ & $\begin{array}{l}\text { Health Care } \\
\text { System } \\
\text { Factors }\end{array}$ & $\begin{array}{c}\text { Socioeconomic } \\
\text { Factors }\end{array}$ \\
\hline $\begin{array}{l}\text { Harrison } \\
\text { et al. }(2013)^{28}\end{array}$ & $\begin{array}{l}\text { United } \\
\text { States }\end{array}$ & 98 & $\begin{array}{l}\text { Patients aged } \\
\geq 24 \text { years, } \\
\text { with no record } \\
\text { of redeeming } \\
\text { a new statin } \\
\text { medication } \\
\text { within } 1 \text { to } 2 \\
\text { weeks of being } \\
\text { ordered }\end{array}$ & $\begin{array}{l}\text { Phone inter- } \\
\text { view data; } \\
\text { self-reported } \\
\text { nonadherence }\end{array}$ & $\begin{array}{l}\text { Concerns about } \\
\text { taking the } \\
\text { medication } \\
\text { Patients' prefer- } \\
\text { ence for life- } \\
\text { style modifica- } \\
\text { tion (e.g., diet } \\
\text { and exercise) } \\
\text { instead of tak- } \\
\text { ing medication } \\
\text { Fear of side } \\
\text { effects } \\
\text { Patients' per- } \\
\text { ceived redun- } \\
\text { dancy and inef- } \\
\text { fectiveness of } \\
\text { medication }\end{array}$ & - & $\begin{array}{l}\text { Lack of } \\
\text { communication } \\
\text { between patient } \\
\text { and physician }\end{array}$ & - & $\begin{array}{l}\text { Financial } \\
\text { hardships } \\
\text { Inadequate } \\
\text { health literacy }\end{array}$ \\
\hline $\begin{array}{l}\text { Jackevicius } \\
\text { et al. }(2008)^{19}\end{array}$ & Canada & 4,591 & $\begin{array}{l}\text { Acute myocar- } \\
\text { dial infarction } \\
\text { (AMI) patients } \\
\text { aged } \geq 66 \text { years, } \\
\text { enrolled in } \\
\text { the Enhanced } \\
\text { Feedback } \\
\text { for Effective } \\
\text { Cardiac } \\
\text { Treatment } \\
\text { (EFFECT) } \\
\text { study registry }\end{array}$ & $\begin{array}{l}\text { The EFFECT } \\
\text { study registry } \\
\text { and the AMI } \\
\text { charts it col- } \\
\text { lects from } 104 \\
\text { acute care } \\
\text { hospitals in } \\
\text { Ontario }\end{array}$ & $\begin{array}{l}\text { Patients' } \\
\text { perceived } \\
\text { ineffectiveness } \\
\text { and } \\
\text { redundancy of } \\
\text { medication } \\
\text { Older patients }\end{array}$ & $\begin{array}{l}\text { Patients with } \\
\text { more pre- } \\
\text { AMI/baseline } \\
\text { prescriptions }\end{array}$ & $\begin{array}{l}\text { Patients who } \\
\text { do not receive } \\
\text { medication } \\
\text { counseling and } \\
\text { education after } \\
\text { discharge } \\
\text { Patients who do } \\
\text { not have a car- } \\
\text { diologist as the } \\
\text { most respon- } \\
\text { sible physician }\end{array}$ & - & - \\
\hline $\begin{array}{l}\text { Jackson } \\
\text { et al. }(2014)^{16}\end{array}$ & $\begin{array}{l}\text { United } \\
\text { States }\end{array}$ & 29,238 & $\begin{array}{l}\text { Adult patients } \\
\text { aged } \geq 18 \\
\text { years, with a } \\
\text { new electronic } \\
\text { prescription } \\
\text { for medications } \\
\text { intended to } \\
\text { treat chronic } \\
\text { conditions, as } \\
\text { supplied by } \\
\text { the Pharmacy } \\
\text { Quality } \\
\text { Alliance (PQA) }\end{array}$ & $\begin{array}{l}\text { Prescription } \\
\text { data from } \\
100 \text { retail } \\
\text { pharmacies }\end{array}$ & $\begin{array}{l}\text { Slightly } \\
\text { younger in age: } \\
59.42 \text { vs. } 59.60 \\
\text { years }\end{array}$ & $\begin{array}{l}\text { PQA-defined } \\
\text { drug class } \\
\text { (e.g., high } \\
\text { nonadherence } \\
\text { is observed for } \\
\text { antiretrovirals) } \\
\text { Higher out-of- } \\
\text { pocket costs for } \\
\text { medication } \\
\text { Prescriptions } \\
\text { accompanied } \\
\text { by another pre- } \\
\text { scription on the } \\
\text { same day }\end{array}$ & $\begin{array}{l}\text { Higher nonad- } \\
\text { herence when } \\
\text { prescriber is } \\
\text { neither a phy- } \\
\text { sician (both } \\
\text { specialist and } \\
\text { primary care), } \\
\text { physician } \\
\text { assistant, or } \\
\text { advanced prac- } \\
\text { tice nurse } \\
\text { Nonadherence } \\
\text { more likely to } \\
\text { occur in phar- } \\
\text { macies with } \\
\text { lower prescrip- } \\
\text { tion volumes }\end{array}$ & - & $\begin{array}{l}\text { Higher nonad- } \\
\text { herence when } \\
\text { prescriptions } \\
\text { originate in } \\
\text { pharmacies } \\
\text { located in } \\
\text { neighborhoods } \\
\text { with higher } \\
\text { household } \\
\text { incomes and } \\
\text { educational } \\
\text { levels }\end{array}$ \\
\hline
\end{tabular}


TABLE 1 Studies Included in Systematic Review (continued)

\begin{tabular}{|c|c|c|c|c|c|c|c|c|c|}
\hline \multirow[b]{2}{*}{ Source } & \multirow[b]{2}{*}{ Country } & \multicolumn{2}{|c|}{ Participant Profile } & \multirow[b]{2}{*}{$\begin{array}{c}\text { How } \\
\text { Adherence Is } \\
\text { Assessed }\end{array}$} & \multicolumn{5}{|c|}{ Main Factors Addressed for Primary Nonadherence } \\
\hline & & $\begin{array}{c}\text { Final } \\
\text { Sample } \\
\text { Size }\end{array}$ & $\begin{array}{c}\text { Sample } \\
\text { Description }\end{array}$ & & $\begin{array}{l}\text { Patient } \\
\text { Factors }\end{array}$ & $\begin{array}{l}\text { Medication } \\
\text { Factors }\end{array}$ & $\begin{array}{c}\text { Health Care } \\
\text { Provider } \\
\text { Factors }\end{array}$ & $\begin{array}{c}\text { Health Care } \\
\text { System } \\
\text { Factors }\end{array}$ & $\begin{array}{c}\text { Socioeconomic } \\
\text { Factors }\end{array}$ \\
\hline $\begin{array}{l}\text { Karter } \\
\text { et al. }(2010)^{29}\end{array}$ & $\begin{array}{l}\text { United } \\
\text { States }\end{array}$ & 169 & $\begin{array}{l}\text { Patients with } \\
\text { type } 2 \text { diabetes } \\
\text { receiving a } \\
\text { new electronic } \\
\text { prescription for } \\
\text { insulin-those } \\
\text { who are pri- } \\
\text { mary adherent } \\
\text { and primary } \\
\text { nonadherent }\end{array}$ & $\begin{array}{l}\text { Data from com- } \\
\text { puter-assisted } \\
\text { interviews and } \\
\text { self-adminis- } \\
\text { tered mailed } \\
\text { surveys }\end{array}$ & $\begin{array}{l}\text { Patients' } \\
\text { decision } \\
\text { to improve } \\
\text { other health } \\
\text { behaviors } \\
\text { instead of } \\
\text { insulin-taking } \\
\text { Patients' } \\
\text { perceived } \\
\text { negative impact } \\
\text { on social and } \\
\text { work life } \\
\text { Injection } \\
\text { phobia } \\
\text { Concerns about } \\
\text { side effects }\end{array}$ & - & $\begin{array}{l}\text { Lack of pro- } \\
\text { vider-patient } \\
\text { communication } \\
\text { and explana- } \\
\text { tion of the } \\
\text { potential risks } \\
\text { and benefits } \\
\text { associated with } \\
\text { insulin } \\
\text { Inadequate } \\
\text { shared } \\
\text { decision } \\
\text { making } \\
\text { between } \\
\text { provider and } \\
\text { patient } \\
\text { Lack of insulin } \\
\text { self-treatment } \\
\text { training for } \\
\text { patients }\end{array}$ & - & - \\
\hline $\begin{array}{l}\text { Polinski } \\
\text { et al. }(2014)^{25}\end{array}$ & $\begin{array}{l}\text { United } \\
\text { States }\end{array}$ & 26 & $\begin{array}{l}\text { Patients aged } \\
\geq 25 \text { years with } \\
\text { PNA for anti- } \\
\text { hypertensive } \\
\text { medications }\end{array}$ & $\begin{array}{l}\text { Focus group } \\
\text { discussions }\end{array}$ & $\begin{array}{l}\text { Patients' } \\
\text { misperception } \\
\text { about } \\
\text { medication } \\
\text { Fear of side } \\
\text { effects } \\
\text { Patients' dis- } \\
\text { trust of health } \\
\text { care provider } \\
\text { Suspicion of } \\
\text { provider's } \\
\text { diagnosis and } \\
\text { motivation to } \\
\text { prescribe }\end{array}$ & $\begin{array}{l}\text { Cost } \\
\text { Complexity } \\
\text { of medication } \\
\text { regimen } \\
\text { (polypharmacy) }\end{array}$ & $\begin{array}{l}\text { Poor communi- } \\
\text { cation between } \\
\text { physician and } \\
\text { patient }\end{array}$ & - & - \\
\hline $\begin{array}{l}\text { Pottegård } \\
\text { et al. (2014)8 }\end{array}$ & Denmark & 146,959 & $\begin{array}{l}\text { Patients aged } \\
\geq 18 \text { years } \\
\text { with free and } \\
\text { direct access } \\
\text { to general } \\
\text { practitioners }\end{array}$ & $\begin{array}{l}\text { Pharmacy } \\
\text { records and } \\
\text { prescription } \\
\text { registry data }\end{array}$ & $\begin{array}{l}\text { Female } \\
\text { Younger } \\
\text { patients aged } \\
\text { 18-29 years } \\
\text { Patients with } \\
\text { a diagnosis of } \\
\text { ischemic heart } \\
\text { disease are less } \\
\text { adherent } \\
\text { Patients with } \\
\text { a diagnosis } \\
\text { of chronic } \\
\text { obstructive } \\
\text { pulmonary } \\
\text { disease are } \\
\text { more adherent }\end{array}$ & Polypharmacy & - & - & $\begin{array}{l}\text { Highest PNA } \\
\text { among patients } \\
\text { who earn } \\
<250,000 \\
\text { Danish krone } \\
\text { per year }\end{array}$ \\
\hline
\end{tabular}

continued on next page 
TABLE 1 Studies Included in Systematic Review (continued)

\begin{tabular}{|c|c|c|c|c|c|c|c|c|c|}
\hline \multirow[b]{2}{*}{ Source } & \multirow[b]{2}{*}{ Country } & \multicolumn{2}{|c|}{ Participant Profile } & \multirow[b]{2}{*}{$\begin{array}{c}\text { How } \\
\text { Adherence Is } \\
\text { Assessed }\end{array}$} & \multicolumn{5}{|c|}{ Main Factors Addressed for Primary Nonadherence } \\
\hline & & $\begin{array}{l}\text { Final } \\
\text { Sample } \\
\text { Size }\end{array}$ & $\begin{array}{c}\text { Sample } \\
\text { Description }\end{array}$ & & $\begin{array}{l}\text { Patient } \\
\text { Factors }\end{array}$ & $\begin{array}{l}\text { Medication } \\
\text { Factors }\end{array}$ & $\begin{array}{c}\text { Health Care } \\
\text { Provider } \\
\text { Factors }\end{array}$ & $\begin{array}{l}\text { Health Care } \\
\text { System } \\
\text { Factors }\end{array}$ & $\begin{array}{c}\text { Socioeconomic } \\
\text { Factors }\end{array}$ \\
\hline $\begin{array}{l}\text { Raebel } \\
\text { et al. }(2011)^{7}\end{array}$ & $\begin{array}{l}\text { United } \\
\text { States }\end{array}$ & 12,061 & $\begin{array}{l}\text { Members of } \\
\text { an integrated } \\
\text { health delivery } \\
\text { system, with a } \\
\text { newly initiated } \\
\text { order for an } \\
\text { antihyperten- } \\
\text { sive, antidia- } \\
\text { betic, or anti- } \\
\text { hyperlipidemic } \\
\text { medication }\end{array}$ & $\begin{array}{l}\text { Electronic } \\
\text { health records } \\
\text { within an inte- } \\
\text { grated system }\end{array}$ & - & $\begin{array}{l}\text { PNA varied } \\
\text { by therapeutic } \\
\text { class-highest } \\
\text { among those } \\
\text { who ordered } \\
\text { antihyperlip- } \\
\text { idemic medi- } \\
\text { cations and } \\
\text { lowest among } \\
\text { those who } \\
\text { ordered anti- } \\
\text { hypertensive } \\
\text { medications }\end{array}$ & $\begin{array}{l}\text { PNA lower } \\
\text { for antihy- } \\
\text { perlipidemic } \\
\text { medications } \\
\text { prescribed by } \\
\text { a provider in } \\
\text { a nonprimary } \\
\text { care } \\
\text { department }\end{array}$ & - & - \\
\hline $\begin{array}{l}\text { Rashid } \\
\text { et al. }(2017)^{17}\end{array}$ & $\begin{array}{l}\text { United } \\
\text { States }\end{array}$ & 9,050 & $\begin{array}{l}\text { Patients aged } \\
\geq 18 \text { years with } \\
\text { new overactive } \\
\text { bladder } \\
\text { prescriptions }\end{array}$ & $\begin{array}{l}\text { Electronic } \\
\text { medical } \\
\text { records }\end{array}$ & $\begin{array}{l}\text { Female } \\
\text { Younger } \\
\text { patients aged } \\
56.9 \text { years on } \\
\text { average, com- } \\
\text { pared with the } \\
\text { overall average } \\
\text { age of } 62.6 \\
\text { years } \\
\text { Patients who } \\
\text { have fewer } \\
\text { comorbid } \\
\text { conditions } \\
\text { (i.e., generally } \\
\text { healthier } \\
\text { otherwise) } \\
\text { Race other than } \\
\text { white }\end{array}$ & $\begin{array}{l}\text { Patients who } \\
\text { have fewer con- } \\
\text { comitant medi- } \\
\text { cations and } \\
\text { prescriptions } \\
\text { dispensed in } \\
\text { the past year }\end{array}$ & - & $\begin{array}{l}\text { Patients who } \\
\text { have commer- } \\
\text { cial insurance } \\
\text { compared with } \\
\text { those with } \\
\text { Medicaid and } \\
\text { Medicare }\end{array}$ & - \\
\hline $\begin{array}{l}\text { Reynolds } \\
\text { et al. }(2013)^{30}\end{array}$ & $\begin{array}{l}\text { United } \\
\text { States }\end{array}$ & 8,454 & $\begin{array}{l}\text { Women aged } \\
\geq 55 \text { years with } \\
\text { a new prescrip- } \\
\text { tion of oral } \\
\text { bisphosphonates }\end{array}$ & $\begin{array}{l}\text { Electronic } \\
\text { medical } \\
\text { records }\end{array}$ & $\begin{array}{l}\text { Older women } \\
\text { with prior } \\
\text { emergency } \\
\text { department } \\
\text { visits } \\
\text { Patients' per- } \\
\text { ceived need for } \\
\text { and benefits } \\
\text { and risks asso- } \\
\text { ciated with } \\
\text { medication }\end{array}$ & - & $\begin{array}{l}\text { Prescriptions } \\
\text { written by pro- } \\
\text { viders with } 10 \\
\text { or more years } \\
\text { of experience } \\
\text { more likely to } \\
\text { be redeemed }\end{array}$ & - & - \\
\hline
\end{tabular}


TABLE 1 Studies Included in Systematic Review (continued)

\begin{tabular}{|c|c|c|c|c|c|c|c|c|c|}
\hline \multirow[b]{2}{*}{ Source } & \multirow[b]{2}{*}{ Country } & \multicolumn{2}{|c|}{ Participant Profile } & \multirow[b]{2}{*}{$\begin{array}{c}\text { How } \\
\text { Adherence Is } \\
\text { Assessed }\end{array}$} & \multicolumn{5}{|c|}{ Main Factors Addressed for Primary Nonadherence } \\
\hline & & $\begin{array}{c}\text { Final } \\
\text { Sample } \\
\text { Size }\end{array}$ & $\begin{array}{c}\text { Sample } \\
\text { Description }\end{array}$ & & $\begin{array}{l}\text { Patient } \\
\text { Factors }\end{array}$ & $\begin{array}{l}\text { Medication } \\
\text { Factors }\end{array}$ & $\begin{array}{c}\text { Health Care } \\
\text { Provider } \\
\text { Factors }\end{array}$ & $\begin{array}{c}\text { Health Care } \\
\text { System } \\
\text { Factors }\end{array}$ & $\begin{array}{c}\text { Socioeconomic } \\
\text { Factors }\end{array}$ \\
\hline $\begin{array}{l}\text { Shin } \\
\text { et al. }(2012)^{5}\end{array}$ & $\begin{array}{l}\text { United } \\
\text { States }\end{array}$ & $\begin{array}{c}569,095 \\
\text { new pre- } \\
\text { scriptions } \\
(398,025 \\
\text { patients) }\end{array}$ & $\begin{array}{l}\text { New prescrip- } \\
\text { tions written } \\
\text { for } 10 \text { thera- } \\
\text { peutic drug } \\
\text { groups in a } \\
\text { 3-month } \\
\text { period }\end{array}$ & $\begin{array}{l}\text { Electronic } \\
\text { medical records }\end{array}$ & $\begin{array}{l}\text { Black and } \\
\text { Hispanic } \\
\text { patients } \\
\text { Patients naive } \\
\text { to therapy and } \\
\text { treatment } \\
\text { Patients with } \\
\text { baseline } \\
\text { comorbidities } \\
\text { Patients who } \\
\text { redeem at least } \\
\text { l prescription } \\
\text { in the previous } \\
\text { year or had a } \\
\text { prescription for } \\
\text { symptomatic } \\
\text { disease } \\
\text { Younger } \\
\text { patients more } \\
\text { likely to fill } \\
\text { acute medica- } \\
\text { tion; less likely } \\
\text { for chronic } \\
\text { medication }\end{array}$ & $\begin{array}{l}\text { Types of drug } \\
\text { group } \\
\text { Regimen } \\
\text { complexity } \\
\text { Cost of } \\
\text { medication } \\
\text { (only when } \\
\text { disease is } \\
\text { acute) }\end{array}$ & - & - & - \\
\hline $\begin{array}{l}\text { Storm } \\
\text { et al. }(2008)^{21}\end{array}$ & Denmark & 322 & $\begin{array}{l}\text { Outpatients of } \\
\text { a public } \\
\text { hospital } \\
\text { dermatology } \\
\text { department, } \\
\text { who receive a } \\
\text { prescription } \\
\text { for an initial } \\
\text { treatment with } \\
\text { a previously } \\
\text { untried } \\
\text { medication }\end{array}$ & $\begin{array}{l}\text { Electronic } \\
\text { pharmacy } \\
\text { register }\end{array}$ & 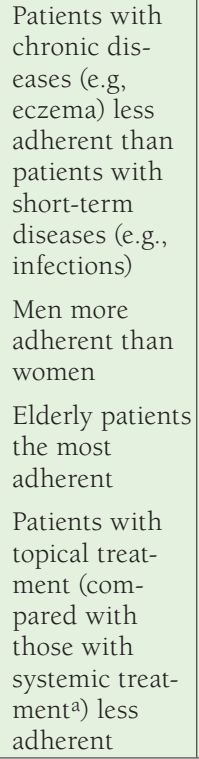 & - & $\begin{array}{l}\text { Better } \\
\text { adherence } \\
\text { among patients } \\
\text { who see } \\
\text { specialists } \\
\text { rather than } \\
\text { junior } \\
\text { physicians }\end{array}$ & - & - \\
\hline
\end{tabular}

continued on next page 
TABLE 1 Studies Included in Systematic Review (continued)

\begin{tabular}{|c|c|c|c|c|c|c|c|c|c|}
\hline \multirow[b]{2}{*}{ Source } & \multirow[b]{2}{*}{ Country } & \multicolumn{2}{|c|}{ Participant Profile } & \multirow[b]{2}{*}{$\begin{array}{c}\text { How } \\
\text { Adherence Is } \\
\text { Assessed }\end{array}$} & \multicolumn{5}{|c|}{ Main Factors Addressed for Primary Nonadherence } \\
\hline & & $\begin{array}{c}\text { Final } \\
\text { Sample } \\
\text { Size } \\
\end{array}$ & $\begin{array}{c}\text { Sample } \\
\text { Description }\end{array}$ & & $\begin{array}{l}\text { Patient } \\
\text { Factors } \\
\end{array}$ & $\begin{array}{c}\text { Medication } \\
\text { Factors } \\
\end{array}$ & $\begin{array}{c}\text { Health Care } \\
\text { Provider } \\
\text { Factors }\end{array}$ & $\begin{array}{c}\text { Health Care } \\
\text { System } \\
\text { Factors }\end{array}$ & $\begin{array}{c}\text { Socioeconomic } \\
\text { Factors }\end{array}$ \\
\hline $\begin{array}{l}\text { Thengilsdóttir } \\
\text { et al. }(2015)^{6}\end{array}$ & Iceland & 10,685 & $\begin{array}{l}\text { Adult patients } \\
\text { aged } \geq 18 \text { years } \\
\text { from the capital } \\
\text { area in Iceland } \\
\text { who received } \\
\text { a new statin or } \\
\text { antidepressant } \\
\text { prescription } \\
\text { within the } \\
\text { study period }\end{array}$ & $\begin{array}{l}\text { Prescription } \\
\text { database } \\
\text { records }\end{array}$ & $\begin{array}{l}\text { Vulnerable } \\
\text { groups of } \\
\text { patients with } \\
\text { disabilities pre- } \\
\text { scribed expen- } \\
\text { sive drugs } \\
\text { Women and } \\
\text { younger } \\
\text { patients }\end{array}$ & $\begin{array}{l}\text { Patients pre- } \\
\text { scribed SNRIs } \\
\text { and atorvas- } \\
\text { tatin compared } \\
\text { with those pre- } \\
\text { scribed SSRIs } \\
\text { and simvastatin }\end{array}$ & - & - & - \\
\hline $\begin{array}{l}\text { Wamala } \\
\text { et al. }(2007)^{31}\end{array}$ & Sweden & 31,895 & $\begin{array}{l}\text { Patients aged } \\
21-84 \text { years } \\
\text { who corre- } \\
\text { sponded with } \\
\text { a physician at } \\
\text { a hospital or } \\
\text { primary care } \\
\text { center within a } \\
\text { 3-month } \\
\text { period }\end{array}$ & $\begin{array}{l}\text { Self-reported } \\
\text { nonadherence } \\
\text { using postal } \\
\text { self-adminis- } \\
\text { tered question- } \\
\text { naire }\end{array}$ & - & - & - & $\begin{array}{l}\text { Sweden's "care } \\
\text { on equal terms" } \\
\text { health policies } \\
\text { (publicly } \\
\text { funded health } \\
\text { care system } \\
\text { and subsidized } \\
\text { medication) } \\
\text { less successful } \\
\text { among socio- } \\
\text { economically } \\
\text { disadvantaged } \\
\text { elderly patients }\end{array}$ & $\begin{array}{l}\text { Patients placed } \\
\text { lower on the } \\
\text { socioeconomic } \\
\text { index, espe- } \\
\text { cially elderly } \\
\text { women }\end{array}$ \\
\hline $\begin{array}{l}\text { Williams } \\
\text { et al. }(2007)^{23}\end{array}$ & $\begin{array}{l}\text { United } \\
\text { States }\end{array}$ & 1,064 & $\begin{array}{l}\text { Asthma } \\
\text { patients aged } \\
5-56 \text { years, } \\
\text { with at least } \\
1 \text { electronic } \\
\text { prescription for } \\
\text { inhaled cortico- } \\
\text { steroids and at } \\
\text { least } 3 \text { months } \\
\text { follow-up after } \\
\text { the prescription }\end{array}$ & $\begin{array}{l}\text { Electronic } \\
\text { prescription } \\
\text { information } \\
\text { and pharmacy } \\
\text { fill data } \\
\end{array}$ & $\begin{array}{l}\text { Low baseline } \\
\text { rescue medica- } \\
\text { tion use } \\
\text { Lower per- } \\
\text { ceived need for } \\
\text { medication } \\
\text { Race and } \\
\text { ethnicity }\end{array}$ & - & $\begin{array}{l}\text { Frequency } \\
\text { of contact } \\
\text { between patient } \\
\text { and physician, } \\
\text { especially } \\
\text { for African } \\
\text { American } \\
\text { patients }\end{array}$ & - & - \\
\hline $\begin{array}{l}\text { Wooldridge } \\
\text { et al. (2016) }\end{array}$ & $\begin{array}{l}\text { United } \\
\text { States }\end{array}$ & 341 & $\begin{array}{l}\text { Adult patients } \\
\text { who were hos- } \\
\text { pitalized for } \\
\text { cardiovascular } \\
\text { events, had } \\
\text { new discharge } \\
\text { prescriptions } \\
\text { to fill post- } \\
\text { discharge, and } \\
\text { had received } \\
\text { study interven- } \\
\text { tion about fill- } \\
\text { ing discharge } \\
\text { prescriptions }\end{array}$ & $\begin{array}{l}\text { Secondary } \\
\text { analysis of data } \\
\text { from a random- } \\
\text { ized, controlled } \\
\text { trial evaluating } \\
\text { the effect of tai- } \\
\text { lored interven- } \\
\text { tion in adults } \\
\text { hospitalized for } \\
\text { acute coronary } \\
\text { syndromes or } \\
\text { acute decom- } \\
\text { pensated heart } \\
\text { failure }\end{array}$ & $\begin{array}{l}\text { Single marital } \\
\text { status }\end{array}$ & $\begin{array}{l}\text { Polypharmacy } \\
\text { (having more } \\
\text { than } 10 \text { total } \\
\text { discharge } \\
\text { medications) }\end{array}$ & $\begin{array}{l}\text { Not applicable } \\
\text { as patients have } \\
\text { undergone tai- } \\
\text { lored interven- } \\
\text { tion of phar- } \\
\text { macy-assisted } \\
\text { medication } \\
\text { reconcilia- } \\
\text { tion, discharge } \\
\text { counseling, } \\
\text { low-literacy } \\
\text { adherence aids, } \\
\text { and follow-up } \\
\text { phone calls }\end{array}$ & - & $\begin{array}{l}\text { Low income: } \\
\text { inability to } \\
\text { afford medica- } \\
\text { tion cost and } \\
\text { also faced } \\
\text { transportation } \\
\text { limitations }\end{array}$ \\
\hline
\end{tabular}


TABLE 1 Studies Included in Systematic Review (continued)

\begin{tabular}{|c|c|c|c|c|c|c|c|c|c|}
\hline \multirow[b]{2}{*}{ Source } & \multirow[b]{2}{*}{ Country } & \multicolumn{2}{|c|}{ Participant Profile } & \multirow[b]{2}{*}{$\begin{array}{c}\text { How } \\
\text { Adherence Is } \\
\text { Assessed }\end{array}$} & \multicolumn{5}{|c|}{ Main Factors Addressed for Primary Nonadherence } \\
\hline & & $\begin{array}{l}\text { Final } \\
\text { Sample } \\
\text { Size }\end{array}$ & $\begin{array}{c}\text { Sample } \\
\text { Description }\end{array}$ & & $\begin{array}{l}\text { Patient } \\
\text { Factors }\end{array}$ & $\begin{array}{l}\text { Medication } \\
\text { Factors }\end{array}$ & $\begin{array}{l}\text { Health Care } \\
\text { Provider } \\
\text { Factors }\end{array}$ & $\begin{array}{l}\text { Health Care } \\
\text { System } \\
\text { Factors }\end{array}$ & $\begin{array}{c}\text { Socioeconomic } \\
\text { Factors }\end{array}$ \\
\hline $\begin{array}{l}\text { Wroth } \\
\text { et al. }(2006)^{18}\end{array}$ & $\begin{array}{c}\text { United } \\
\text { States }\end{array}$ & 3,926 & $\begin{array}{l}\text { Adult aged } \geq 18 \\
\text { years, having } \\
\text { lived in the } \\
\text { southeastern } \\
\text { rural commu- } \\
\text { nity for more } \\
\text { than l year, } \\
\text { and visited a } \\
\text { health care } \\
\text { provider in the } \\
\text { previous year }\end{array}$ & $\begin{array}{l}\text { Phone survey } \\
\text { data; self- } \\
\text { reported non- } \\
\text { adherence }\end{array}$ & $\begin{array}{l}\text { Patients aged } \\
<65 \text { years } \\
\text { African } \\
\text { American } \\
\text { Patients with } \\
\text { transportation } \\
\text { problems } \\
\text { Trust and } \\
\text { confidence } \\
\text { in patient- } \\
\text { physician } \\
\text { relationship } \\
\text { Patients' sat- } \\
\text { isfaction with } \\
\text { care provided } \\
\end{array}$ & - & $\begin{array}{l}\text { Trust and } \\
\text { confidence } \\
\text { in patient- } \\
\text { physician } \\
\text { relationship } \\
\text { Patients' sat- } \\
\text { isfaction with } \\
\text { care provided } \\
\text { Patient- } \\
\text { physician } \\
\text { concordance on } \\
\text { medication }\end{array}$ & - & $\begin{array}{l}\text { Annual income } \\
\text { of }<\$ 25,000\end{array}$ \\
\hline $\begin{array}{l}\mathrm{Yu} \\
\text { et al. }(2015)^{26}\end{array}$ & $\begin{array}{l}\text { United } \\
\text { States }\end{array}$ & 430 & $\begin{array}{l}\text { Women aged } \\
\geq 55 \text { years with } \\
\text { an untreated } \\
\text { osteoporosis } \\
\text { diagnosis (i.e., } \\
\text { no claims for } \\
\text { osteoporosis- } \\
\text { specific } \\
\text { medication) }\end{array}$ & $\begin{array}{c}\text { Mail survey } \\
\text { data }\end{array}$ & $\begin{array}{l}\text { Concern over } \\
\text { side effects of } \\
\text { medication } \\
\text { Patients' beliefs } \\
\text { about osteo- } \\
\text { porosis and } \\
\text { osteoporosis } \\
\text { medication }\end{array}$ & - & - & $\begin{array}{l}\text { Cost of medica- } \\
\text { tion: contribu- } \\
\text { tion of insur- } \\
\text { ance to medica- } \\
\text { tion costs }\end{array}$ & - \\
\hline
\end{tabular}

reason for PNA. In some articles, this was associated with health care provider factors, which will be elaborated in the following subsection.

Demographics that were found to affect primary medication adherence varied from study to study. For example, though most studies found younger patients to be more nonadherent compared with older ones, ${ }^{6,8,14-18}$ Jackevicius et al. (2008) noted the reverse in acute myocardial infarction patients aged 66 and above. ${ }^{19}$ Women were also consistently found to be more nonadherent compared with men. ${ }^{6,8,17,20,21}$ In terms of race/ethnicity, it was largely observed that minority groups in the United States, like Hispanic and African American patients, exhibited higher PNA compared with white patients. ${ }^{5,17,18,22,23}$ One exception was a study of new dermatology patients by Adamson et al. (2017), where Hispanic patients were found to be the most adherent. $^{14}$

Patient health literacy was also directly associated with PNA, where poor health literacy or health literacy limitations yielded higher PNA among patients. ${ }^{22}$ However, a few articles attributed health literacy to health care provider factor, which will be expounded in further detail.

\section{Medication Factors}

Many articles reported that drug regimen constituted a main determinant of PNA, where polypharmacy and complex dosing regimen were identified as the predominant factors. PNA was higher when there were more baseline medications or drugs prescribed concurrently. ${ }^{14-16,19,24}$ In some cases, the complexity of one's overall medication regimen also further acts as a deterrent to adherence. ${ }^{5,25}$ However, Rashid et al. (2017) found that the reverse of polypharmacy applied to a group of patients with overactive bladder prescriptions, where nonadherent patients were those with fewer concomitant medications. ${ }^{17}$

High cost of medication was also identified as a contributor to PNA., ${ }^{54,16,26}$ Drug types were found to influence PNA as well, notably in comparative cases where different drug types were studied. 5,6,16,20,27 For instance, in a study of Portuguese patients with chronic medical conditions, those prescribed antidiabetic drugs were less adherent compared with those given antihypertensive or antihyperlipidemic drugs. ${ }^{20}$ Another medication factor in PNA was related to drug handling, where patients commonly cited having leftover medications at home as a reason to be nonadherent. Da Costa et al. (2015) said this 
could be attributed to medication sharing, medication saving, skipping doses, or different sizes of medication packaging. ${ }^{20}$

\section{Health Care Provider Factors}

Health care provider factors include patient-physician relationship (communication, trust, shared decision making), counseling and education, as well as the experience and credibility of health care providers. In most cases, PNA was attributed to a lack of communication between the patient and physician. ${ }^{18,23,25,28,29}$ For instance, Polinski et al. (2014) found that poor patient-physician communication caused patients' distrust in the health care provider and suspicion in the provider's diagnosis and motivation to prescribe. ${ }^{25}$ Wroth and Pathman (2006) similarly noted that when patients were made to feel cared for, welcome, and comfortable by the health care provider (physician, nurse, office staff), they were more likely to fill prescriptions. ${ }^{18}$

Most studies also discovered a strong relationship between PNA and the experience or credibility of the patient's health care provider. Storm et al. (2008), in their study of Danish outpatients in a public hospital dermatology department, documented better adherence among patients who saw specialists compared with junior physicians. ${ }^{21}$ In terms of experience, prescriptions that were written by providers with 10 or more years of experience were more likely to be filled. ${ }^{30}$ However, Jackson et al. (2014) noted the reverse where PNA was higher among patients receiving prescriptions from primary care physicians relative to physician assistants and advanced practice nurses. ${ }^{16}$

\section{Health Care System Factors}

Four out of the 22 articles included in this review addressed health care system factors in the assessment of PNA. On a national policy level, Wamala et al. (2007) found that Sweden's National Public Health Policy, though implemented to promote equal access to health care, has been less successful among socioeconomically disadvantaged elderly patients. ${ }^{31}$ Despite having subsidized and even free prescribed medication, PNA was still associated with socioeconomic disadvantage-with threefold increased odds among elderly men and a 6-fold increase among women aged 65-84 years. On a health care facility level, Adamson et al. noted an overall decrease in PNA (47\% reduction) with electronic prescriptions as compared with paper prescriptions at a hospital outpatient dermatology clinic, though it was also found that patients with paper prescriptions showed higher adherence during the first 4 days after their index visit. ${ }^{14}$ In addition, the hospital's infrastructure to accommodate Spanish-speaking patients contributed to a higher rate of adherence among its Hispanic patients-an anomaly to the trend in other articles where they exhibited higher PNA compared with white patients.

\section{Socioeconomic Factors}

Financial hardship and income level were largely identified as barriers to primary medication adherence. $8,18,20,24,28,31$ In some studies, financial problems were also directly associated and overlapped with patient and medication factors. For instance, not only was PNA higher among patients placed lower on the socioeconomic index, it was also more significant in elderly women ${ }^{31}$ and in elderly patients who had difficulty affording a high cost of medication. ${ }^{14}$ Wooldridge et al. (2016) said patients with low earnings also faced transportation limitations, which made it challenging for them to commute to the pharmacy to fill prescriptions after discharge. ${ }^{24}$ In contrast to these prevailing findings, Jackson et al. observed higher PNA when prescriptions originated in pharmacies located in neighborhoods with higher household incomes and educational levels. ${ }^{16}$

\section{Metric Analysis}

Figure 2 shows the bibliometric outreach of the studies on PNA. The study from Jackevicius et al. had the most citations, with a total of 230, 171 of which were from QS top universities. ${ }^{19}$ Storm et al. and Karter et al. (2010) also received a large number of citations, with 111 (90 QS) and 107 (94 QS), respectively. ${ }^{21,29}$ Most of these citations were from the field of medicine.

Although some newer studies such as Adamson et al., Rashid et al., and Wooldridge et al. did not have citations, ${ }^{14,17,24}$ they had an altmetrics presence, as illustrated in Figure 3. The study by Adamson et al. had the highest Altmetric Attention Score of 117 , despite only being published recently. ${ }^{14}$ With this surprisingly high score, it belonged in the top $5 \%$ of all research outputs scored by Altmetric.com and had a high attention score (86th percentile) compared with outputs of the same age and source (according to the contextual information provided by Altmetric.com). This can be attributed to the 13 news stories found on 12 news outlets about this study, which included an interview with the study's first author on the MedicalResearch.com blog. ${ }^{32}$ The study also received attention on Twitter, with 46 tweets from 31 users. This highlights the advantage of altmetrics as a quick and timely measurement of the outreach and impact of newly published works.

Figure 4 shows the PlumX Usage and Capture metrics for the studies on PNA. Fallis et al. (2013) registered a high usage count of 7,578, which was attributed to HTML and PDF views on PLoS, PubMedCentral, and EBSCO. ${ }^{33}$ Polinski et al. and Karter et al. also received high usage counts of 1,880 and 3,920, respectively. ${ }^{25,29}$ Adamson et al. had only 1 usage count. ${ }^{14}$ Capture count should be considered more important than usage count, as usage can be easily increased if the authors publicize the article extensively on social media and direct researchers to click on the shared URL to view the abstract or full text. However, capture count cannot be easily garnered, as researchers need to have had enough interest in an article to bookmark, favorite, or export it, for example. 


\section{FIGURE 2 Overview of the Bibliometric Outreach of PNA Studies}

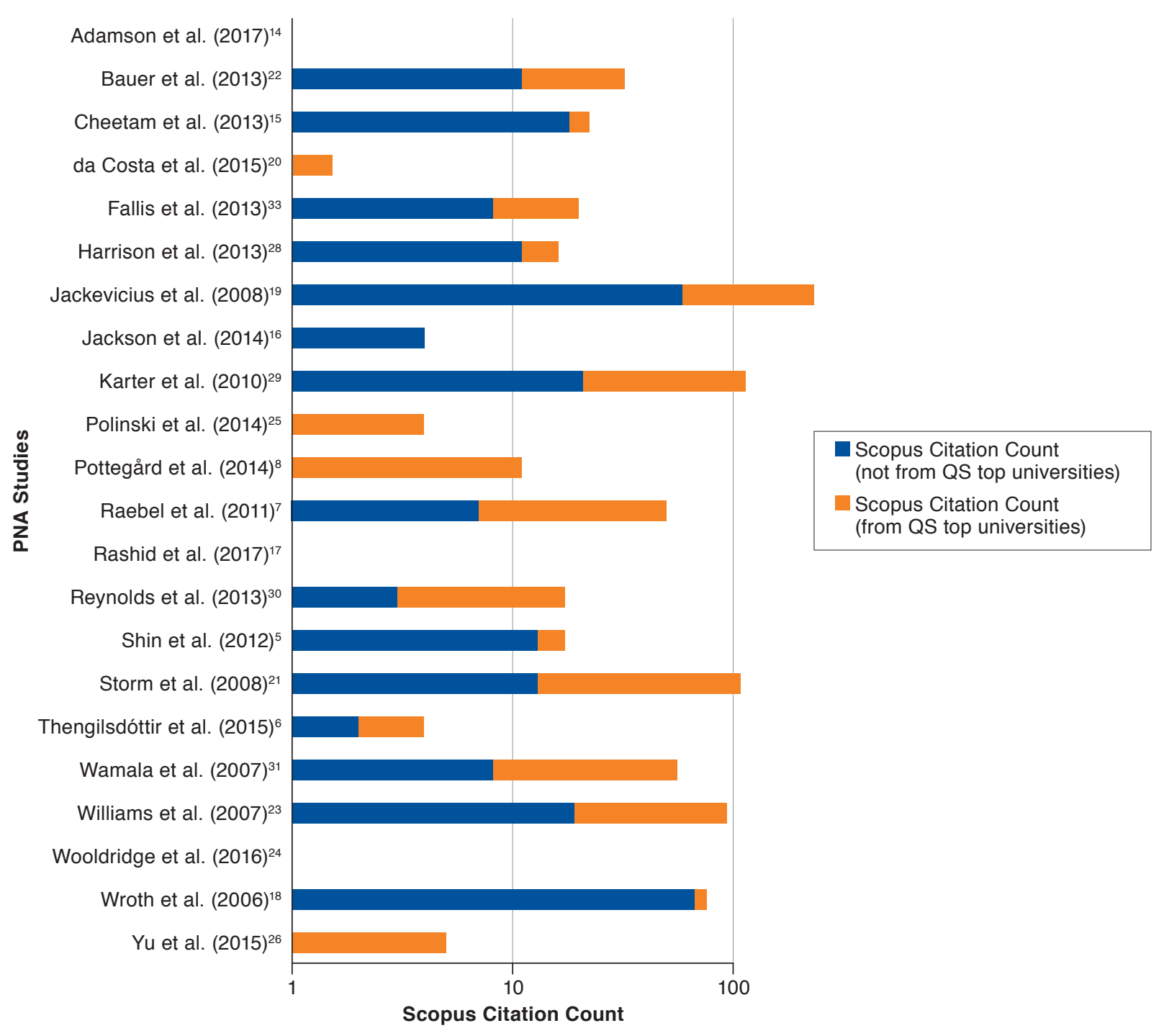

PNA = primary nonadherence; $Q S=2015$ Quacquarelli Symonds university rankings.

\section{Discussion}

Adherence to therapy is fundamental to patient care and constitutes an indispensable process in achieving desired clinical outcomes. Nonadherence can result in poor health outcomes and higher overall health care expenditure. ${ }^{34}$ In the research of medication adherence, extensive literature exists on secondary nonadherence, while PNA has been largely overlooked until recently. Newly developed technology, such as electronic prescribing and electronic medical records, has paved the way for more rigorous, comprehensive, and in-depth study of PNA. PNA is as important to research as secondary nonadherence, and may even be considered more critical as patients fail to initiate treatment by not filling their prescriptions. Furthermore, if a patient fails to possess medication from the beginning, subsequent efforts to improve secondary nonadherence will be futile.

This review classified PNA factors into 5 categories using a previously published approach-patient factors, medication factors, health care provider factors, health care system factors, and socioeconomic factors. Patient factors remained the most highly covered $(n=19)$, addressing a wide range of components such as demographics, beliefs/perceptions/attitudes, health literacy, and medical history. While variables such as demographics (age, sex, and race) and medical history are definitive and unchangeable, 


\section{FIGURE 3 Overview of the Altmetric Outreach of PNA Studies}

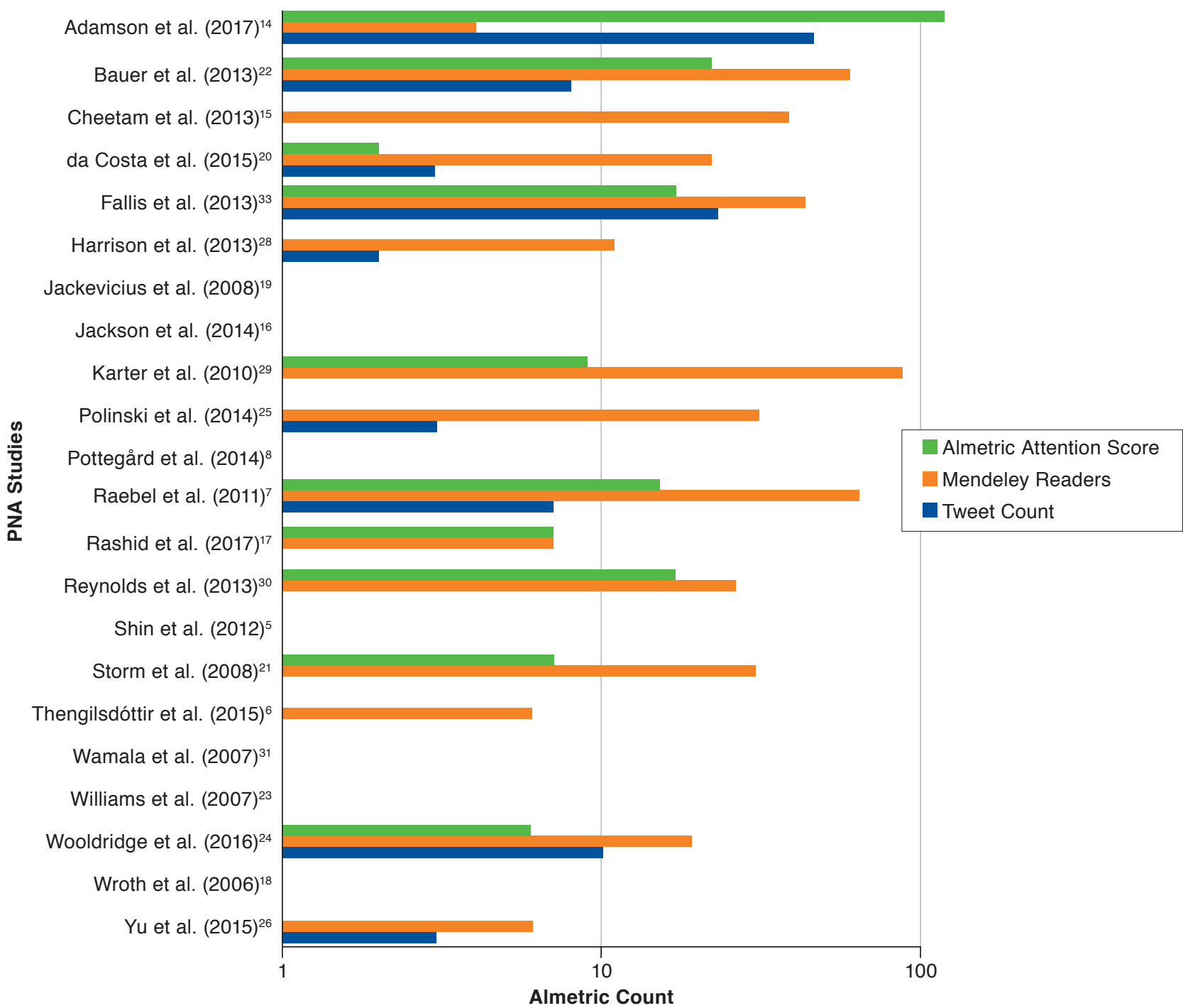

PNA = primary nonadherence .

other patient-related characteristics are potentially modifiable. For instance, a number of articles reported that PNA was largely motivated by patients' perceptions toward the effectiveness, risks, and necessity of their prescribed medication. This represents a modifiable factor, which can be alleviated through various intervention methods that address the root causes of these views. One predominant root cause repeatedly raised in the review is the lack of communication between patient and physician (also classified as a health care provider factor), which contributed significantly to patients filling their medication or cultivated distrust and suspicion in their health care provider. ${ }^{19,25,28,29}$ In this case, interventions like medication counseling or education for patients and closer collaboration between patient and physician can bridge the communication gap and improve primary medication adherence.

Another recurring group of factors in this review of PNA barriers is the health care provider factor $(n=10)$, such as poor patient-physician communication, lack of shared decision making between provider and patient, and the disparity between consulting a specialist and nonspecialist as the main physician. In fact, this review observed that many determinants grouped under the category of health care provider factor overlap or share a causal relationship with the patient 


\section{FIGURE 4 Overview of the PlumX Usage and Capture Counts for PNA Studies}

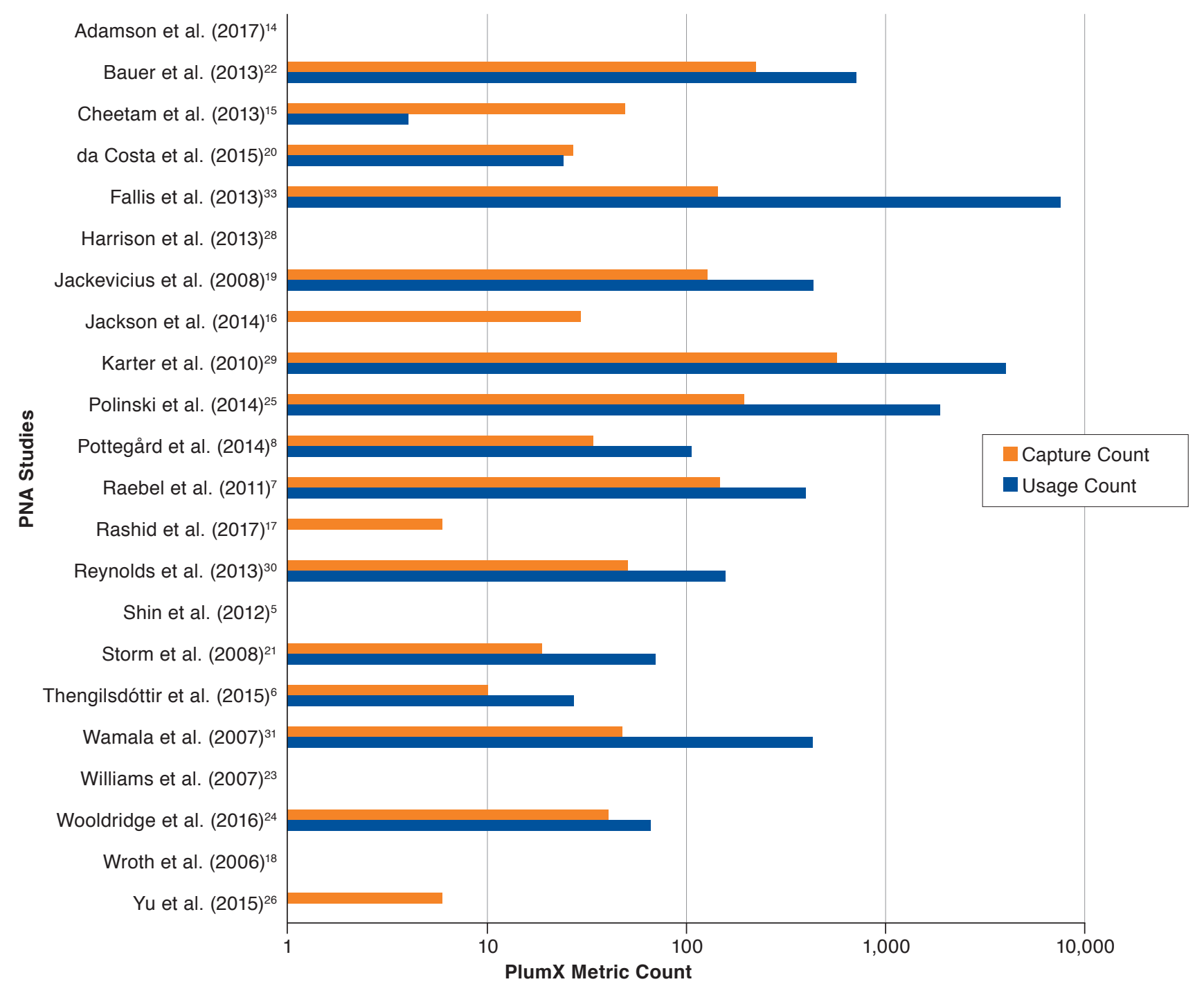

PNA = primary nonadherence.

factors, where one precedes or gives rise to the other. As previously shown, patients' negative perceptions toward a particular drug (patient factor) is largely informed by a lack of communication between them and their physician (health care provider factor). Similarly, inadequate shared decision making between the patient and physician on what medication to prescribe (health care provider factor) also potentially breeds patients' distrust and suspicion in the provider's diagnosis and motivation to prescribe (patient factor), and eventually discourages primary medication adherence. This demonstrates that reasons for PNA, though distinguished in neat categories, are rarely stand-alone causes independent of one another. Instead, they are dynamic and interactive factors, closely associated with each other in a web of interconnectedness. Therefore, given this multidimensional nature of PNA, it is imperative that any solution or intervention method devised is predicated on a comprehensive understanding of the complexity of nonadherence behavior.

While many factors are unchangeable (such as demographics, medical history, and patients' socioeconomic status) or difficult to overcome in the short term due to their large-scale nature (such as the characteristics and operations of a health care system or the cost of medication), some reasons addressed in this review are more fluid and open to intervention at a 
smaller level. These include polypharmacy, patients' views on taking medication, health literacy, patient-physician relationship, and many others. In the existing literature on PNA, there are a number of intervention studies on ways to improve primary medication adherence. A majority of these studies seek to address patients' concerns about medication effect, as well as their lack of information and knowledge about prescribed therapy and its associated benefits, via intervention methods such as voice response phone calls, follow-up letters, and postdischarge pharmacist counseling. ${ }^{4,35-37}$ Most studies reported a positive effect in lowering the rate of PNA. However, there is no known intervention study on other potentially modifiable PNA barriers such as polypharmacy and patient-physician relationship. Future studies could focus on addressing these factors in improving primary medication adherence.

Through our bibliometric and altmetric analysis, we found that $86 \%$ of the articles included in this review had citation counts, while $64 \%$ received attention on social media. This finding provides a clear indication that studies on PNA continue to receive a fair amount of attention from the research community, as well as on social media and in the news. This finding also presents an optimistic sign that research on PNA, long sidelined in the study of medication nonadherence, is beginning to gain more importance in recent years. Its increasing presence on social media and online news also signifies a growing awareness of the issue. This might introduce new perspectives to the costly phenomenon of medication nonadherence, and subsequently, initiate more explorations on intervention methods to minimize PNA and improve the behavior of medication nonadherence in general.

\section{Limitations}

This systematic review contains a few limitations. As articles included in this review were only sourced from 4 specific databases (PubMed, PsycINFO, CINAHL, and ScienceDirect), the search might not have covered all available studies on PNA.

The exclusive topic also yielded few search results, with only 2 studies that produced qualitative research data, which used the methods of phone interview and focus group discussion, respectively. A majority of articles $(n=20)$ were quantitative in nature, with data obtained mostly from patients' electronic medical and pharmacy records. These records captured information that we have categorized as patient and medication factors, such as demographics, medication regimen, and medication cost. As barriers to medication nonadherence are largely psycho-social in nature, PNA behavior cannot be comprehensively understood through the examination of these quantitative data alone. As previously discussed, the multifaceted characteristics of PNA also necessitate a deeper analysis of all factor categories and their shared relationships. The common methodology of assessing adherence in this review (electronic medical and pharmacy records) thus resulted in a skewed focus on the categories of patient and medication factors and implied a neglect of other factor groups that are essential to understanding PNA. Therefore, a major limitation of this review lies in the severe lack of qualitative research on PNA, suggesting that other more complex traits of nonadherence behavior might have been overlooked or are yet to be discovered.

\section{Conclusions}

This systematic review found a multitude of factors behind PNA, which we classified into 5 categories using a multilevel approach. Patient and health care provider factors play a key role in influencing patient decisions to initiate treatment. Those 2 categories share a dynamic relationship and are highly modifiable. As presented, patient attitudes toward taking medication is a primary patient-related barrier to initiating treatment and is often informed by health care providers, which has 2 implications: First, it suggests that any effort to reduce PNA should directly target these factor groups for a more effective outcome; second, it highlights the close association between patients' outlooks and their health care providers, thereby providing a more concrete direction for intervention. As observed from current studies, health care professionals such as nurses and pharmacists actively contribute to minimizing PNA among patients by providing health care information. Therefore, future intervention could continue to boost the effect of health care providers, whether by encouraging greater transparency and shared decision making between physician and patient or by engaging nurses and counselors to help improve patients' health literacy and their perceptions regarding taking medication. Stronger collaboration between patient and health care provider will act as a useful starting point in overcoming the complex and multifaceted behavior of PNA.

Finally, as the current pool of literature on PNA is highly limited, there is a great need for more well-designed studies and qualitative research into this topic and for future intervention studies on PNA to assess the close association between patient and health care provider factors.

\section{Authors}

SHAN-QI LEE, BSOCSc; ARAVIND SESAGIRI RAAMKUMAR, MSc; JINHUI LI, BEng; YUANYUAN CAO, JM; KANOKKORN WITEDWITTAYANUSAT, MA; LUXI CHEN, PhD; and YINLENG THENG, PhD, Centre for Health and Sustainable Cities, Wee Kim Wee School of Communication and Information, Nanyang Technological University, Singapore.

AUTHOR CORRESPONDENCE: Shan-Qi Lee, BSocSc, Centre for Healthy and Sustainable Cities, Wee Kim Wee School of Communication and Information, Nanyang Technological University, 31 Nanyang Link, WKWSCI Bldg., Singapore 637718.

Tel.: 65-69083444; E-mail: sqlee@ntu.edu.sg. 


\section{DISCLOSURES}

This research was supported by the National Research Foundation Singapore under its National Innovation Challenge on Active and Confident Ageing (Award No. MOH/NIC/CAHIG03/2016) and administered by the Singapore Ministry of Health's National Medical Research Council. This research was also supported by the National Research Foundation within the Prime Minister's Office of Singapore, under its Science of Research, Innovation and Enterprise Programme (SRIE Award No. NRF2014-NRF-SRIE001-019). The authors have no relevant conflicts of interest to disclose.

\section{REFERENCES}

1. Yap AF, Thirumoorthy T, Kwan YH. Medication adherence in the elderly. J Clin Gerontol Geriatr. 2016;7(2):64-67.

2. Latif S, McNicoll L. Medication and nonadherence in the older adult. Med Health R I. 2009;92(12):418-19.

3. Adams AJ, Stolpe SF. Defining and measuring primary medication nonadherence: development of a quality measure. J Manag Care Spec Pharm. 2016;22(5):516-23. Available at: https://www.jmcp.org/doi/10.18553/ jmcp.2016.22.5.516.

4. Derose SF, Green K, Marrett E, et al. Automated outreach to increase primary adherence to cholesterol-lowering medications. JAMA Intern Med. 2013;173(1):38-43

5. Shin J, McCombs JS, Sanchez RJ, Udall M, Deminski MC, Cheetham TC. Primary nonadherence to medications in an integrated healthcare setting. Am J Manag Care. 2012;18(8):426-34.

6. Thengilsdottir G, Pottegård A, Linnet K, Halldorsson M, Almarsdottir A, Gardarsdottir H. Do patients initiate therapy? Primary nonadherence to statins and antidepressants in Iceland. Int J Clin Pract. 2015;69(5):597-603.

7. Raebel MA, Carroll NM, Ellis JL, Schroeder EB, Bayliss EA. Importance of including early nonadherence in estimations of medication adherence. Ann Pharmacother. 2011;45(9):1053-60.

8. Pottegård A, Christensen RD, Houji A, et al. Primary nonadherence in general practice: a Danish register study. Eur J Clin Pharmacol. 2014;70(6):757-63.

9. Dalvi V, Mekoth N. Patient nonadherence: an interpretative phenomenological analysis. Int J Health Care Qual Assur. 2017;30(3):274-84.

10. Miller NH, Hill M, Kottke T, Ockene IS. The multilevel compliance challenge: recommendations for a call to action. Circulation. 1997;95(4):1085-90.

11. Haustein S, Larivière V. The use of bibliometrics for assessing research: possibilities, limitations and adverse effects. Incentives and Performance. Switzerland: Springer International Publishing; 2015:121-39.

12. Priem J, Taraborelli D, Groth P, Neylon C. Altmetrics: a manifesto. 2010. Available at: http://altmetrics.org/manifesto/. Accessed June 15, 2018

13. Bornmann L. Do altmetrics point to the broader impact of research? An overview of benefits and disadvantages of altmetrics. J Informetr. 2014;8(4):895-903.

14. Adamson AS, Suarez EA, Gorman AR. Association between method of prescribing and primary nonadherence to dermatologic medication in an urban hospital population. JAMA Dermatol. 2017;153(1):49-54.

15. Cheetham TC, Niu F, Green K, et al. Primary nonadherence to statin medications in a managed care organization. J Manag Care Pharm. 2013;19(5):367-73. Available at: https://www.jmcp.org/doi/10.18553/ jmcp.2013.19.5.367.
16. Jackson TH, Bentley JP, McCaffrey I, et al. Store and prescription characteristics associated with primary medication nonadherence. J Manag Care Pharm. 2014;20(8):824-32. Available at: https://www.jmcp.org/doi/10.18553/ jmcp.2014.20.8.824

17. Rashid N, Vassilakis M, Lin KJ, Kristy R, Ng DB. Primary nonadherence to overactive bladder medications in an integrated managed care health care system. J Manag Care Spec Pharm. 2017;23(4):484-93. Available at: https:// www.jmcp.org/doi/10.18553/jmcp.2017.23.4.484

18. Wroth TH, Pathman DE. Primary medication adherence in a rural population: the role of the patient-physician relationship and satisfaction with care. J Am Board Fam Med. 2006;19(5):478-86.

19. Jackevicius CA, Li P, Tu JV. Prevalence, predictors, and outcomes of primary nonadherence after acute myocardial infarction. Circulation. 2008;117(8):1028-36.

20. da Costa FA, Pedro AR, Teixeira I, Bragança F, Da Silva JA, Cabrita J. Primary nonadherence in Portugal: findings and implications. Int J Clin Pharm. 2015;37(4):626-35.

21. Storm A, Andersen SE, Benfeldt E, Serup J. One in 3 prescriptions are never redeemed: primary nonadherence in an outpatient clinic. J Am Acad Dermatol. 2008;59(1):27-33.

22. Bauer AM, Schillinger D, Parker MM, et al. Health literacy and antidepressant medication adherence among adults with diabetes: the diabetes study of Northern California (DISTANCE). J Gen Intern Med. 2013;28(9):1181-87

23. Williams LK, Joseph CL, Peterson EL, et al. Patients with asthma who do not fill their inhaled corticosteroids: a study of primary nonadherence. J Allergy Clin Immunol. 2007;120(5):1153-59.

24. Wooldridge K, Schnipper JL, Goggins K, Dittus RS, Kripalani S. Refractory primary medication nonadherence: Prevalence and predictors after pharmacist counseling at hospital discharge. J Hosp Med. 2016;11(1):48-51.

25. Polinski J, Kesselheim A, Frolkis J, Wescott P, Allen-Coleman C, Fischer M. A matter of trust: patient barriers to primary medication adherence. Health Educ Res. 2014;29(5):755-63.

26. Yu J, Brenneman SK, Sazonov V, Modi A. Reasons for not initiating osteoporosis therapy among a managed care population. Patient Prefer Adherence. 2015;9:821.

27. Raebel MA, Ellis JL, Carroll NM, et al. Characteristics of patients with primary nonadherence to medications for hypertension, diabetes, and lipid disorders. J Gen Intern Med. 2012;27(1):57-64

28. Harrison TN, Derose SF, Cheetham TC, et al. Primary nonadherence to statin therapy: patients' perceptions. Am J Manag Care. 2013;19(4):133-39.

29. Karter AJ, Subramanian U, Saha C, et al. Barriers to insulin initiation. Diabetes Care. 2010;33(4):733-35.

30. Reynolds K, Muntner P, Cheetham TC, et al. Primary nonadherence to bisphosphonates in an integrated healthcare setting. Osteoporos Int. 2013;24(9):2509-17

31. Wamala S, Merlo J, Bostrom G, Hogstedt C, Agren G. Socioeconomic disadvantage and primary nonadherence with medication in Sweden. Int J Qual Health Care. 2007;19(3):134-40.

32. Adamson AS. Electronic prescriptions more likely to be filled by patients. MedicalResearch.com. October 27, 2016. Available at: https:// medicalresearch.com/author-interviews/electronic-prescriptions-likely-filledpatients/29121/. Accessed June 28, 2018.

33. Fallis BA, Dhalla IA, Klemensberg J, Bell CM. Primary medication nonadherence after discharge from a general internal medicine service. PloS One. 2013;8(5):e61735. 
34. Johnson MJ, Williams M, Marshall ES. Adherent and nonadherent medication-taking in elderly hypertensive patients. Clin Nurs Res. 1999;8(4):318-35

35. Cizmic A, Heilmann R, Milchak J, Riggs C, Billups S. Impact of interactive voice response technology on primary adherence to bisphosphonate therapy: a randomized controlled trial. Osteoporos Int. 2015;26(8):2131-36.

36. Fischer MA, Jones J, Wright E, et al. A randomized telephone intervention trial to reduce primary medication nonadherence. J Manag Care Spec

Pharm. 2015;21(2):124-31. Available at: https://www.jmcp.org/doi/10.18553/ jmcp.2015.21.2.124.
37. Leguelinel-Blache G, Dubois F, Bouvet S, et al. Improving patient's primary medication adherence: the value of pharmaceutical counseling. Medicine. 2015;94(41):e1805. 